\title{
Explaining the Spectra of Cosmic Ray Groups above the Knee by Escape from the Galaxy
}

\author{
G. Giacinti ${ }^{1}$, M. Kachelrieß ${ }^{2}$, and D. V. Semikoz ${ }^{3}$ \\ ${ }^{1}$ University of Oxford, Clarendon Laboratory, Oxford, United Kingdom \\ ${ }^{2}$ Institutt for fysikk, NTNU, Trondheim, Norway and \\ ${ }^{3}$ AstroParticle and Cosmology (APC), Paris, France
}

\begin{abstract}
We investigate the possibility that the cosmic ray (CR) knee is entirely explained by the energydependent CR leakage from the Milky Way. We test this hypothesis calculating the trajectories of individual CRs with energies between $E / Z=10^{14} \mathrm{eV}$ and $10^{17} \mathrm{eV}$ propagating them in the regular and turbulent Galactic magnetic field. We find a knee-like structure of the CR escape time $\tau_{\text {esc }}(E)$ around $E / Z=$ few $\times 10^{15} \mathrm{eV}$ for a coherence length $l_{\mathrm{c}} \simeq 2 \mathrm{pc}$ of the turbulent field, while the decrease of $\tau_{\text {esc }}(E)$ slows down around $E / Z \simeq 10^{16} \mathrm{eV}$ in models with a weak turbulent magnetic field. Assuming that the injection spectra of CR nuclei are power-laws, the resulting CR intensities in such a turbulence are consistent with the energy spectra of CR nuclei determined by KASCADE and KASCADE-Grande. We calculate the resulting CR dipole anisotropy as well as the source rate in this model.
\end{abstract}

Introduction.-The cosmic ray (CR) energy spectrum follows a power-law on more than ten decades in energy. Only a few breaks, such as the knee at $E_{\mathrm{k}} \approx 4 \mathrm{PeV}$, provide possible clues to how CRs propagate and to what their sources are. In addition to this feature, seen in the total CR flux, the elemental composition of the CR flux in the energy range $10^{15}-10^{17} \mathrm{eV}$ is especially useful to constrain theoretical models for the knee. Such data had been missing, but recently the KASCADE-Grande collaboration has extended the measurements of the intensity of individual groups of CR nuclei up to $10^{17} \mathrm{eV}$ [1, 2]. In the future, the IceCube experiment with its IceTop extension will provide additional constraints on the mass composition around the knee [3]. It is therefore timely to compare not only the predictions for the total CR flux and global quantities such as the elongation rate to the experimental data, but also to consider the intensity of individual groups of CR nuclei.

Three competing explanations for the origin of the knee have been advanced: First, there have been speculations that interactions may change in the multi-TeV region, thereby suppressing the CR flux. This possibility is now excluded by the LHC data. Second, the knee may correspond to the maximum rigidity to which the dominant population of Galactic CR sources can accelerate CRs [4]. In a variation of this suggestion, the knee is caused by the maximal energy of a single nearby source such as Monogem [5]. While it is very natural to expect that differences in supernovae types and their environments lead to a distribution of reachable maximal rigidities, this proposal does not predict the exact energy of the knee or the strength of the flux suppression, without a better knowledge of CR confinement and escape [6].

Finally, the knee may be caused by a change of the diffusion properties of charged CRs [7, 8]. For instance, the knee may correspond to the rigidity at which the CR Larmor radius $r_{\mathrm{L}}$ starts to be of the order of the coherence length $l_{\mathrm{c}}$ of the turbulent Galactic magnetic field
(GMF). Thence the behaviors of the CR diffusion coefficient and confinement time change, which in turn would induce a steepening in the spectrum. Both small-angle scattering [7] and Hall diffusion [8] have been proposed as models for the energy dependence of the diffusion coefficient in this regime. This phenomenological approach to $\mathrm{CR}$ escape that describes $\mathrm{CR}$ propagation by diffusion has two major drawbacks: First, the analytical connection between the diffusion tensor and the underlying magnetic field is know only in certain limiting regimes. Second, the diffusion approximation is not justified at the highest energies we are interested in.

The goal of this Letter is therefore to study CR escape from our Galaxy by propagating individual CRs in detailed GMF models. Given a specific GMF model, this approach allows us to predict the position and the shape of the knee for CR nuclei as a function of $E / Z$. We determine three main observables: The time-averaged grammage $X(E)$ traversed by CRs before escape, the time-dependent intensity of CR nuclei at the position of the Sun, and the amplitude $d$ of the dipole anisotropy. Extrapolating $X(E)$ towards its measured value at low energies, we constrain first the strength of the turbulent Galactic magnetic field. Then we use the CR dipole anisotropy as an indication for the transition from Galactic to extragalactic CRs. Finally, we calculate the CR nuclei energy spectra, and compare them to the intensity of groups of CR nuclei determined by KASCADE and KASCADE-Grande, examining the consistency of the proposed scenario. Our results suggest that the turbulent GMF is weaker than in GMF models as [10] and has a small coherence length. Improving our undestanding of the GMF has important impacts on fields outside $\mathrm{CR}$ research as e.g. indirect DM searches, where our results support the use of a large GMF halo and a large diffusion coefficient.

Simulation procedure - For the propagation of CRs in the GMF, we use the code described and tested in [9]. 
For the present work, we have implemented the most recent GMF models [10, 11], but a more important change is the use of a reduced coherence length, $l_{\mathrm{c}}=2 \mathrm{pc}$, for the turbulent field. Such a value is in line with recent measurements of $l_{\mathrm{c}}$ in some regions of the Galactic disk, see e.g. [12]. We model the random field as isotropic Kolmogorov turbulence, $\mathcal{P}(k) \propto k^{-5 / 3}$ extending down to a minimal length scale $l_{\min } \lesssim 1 \mathrm{AU}$.

We assume that the surface density of CR sources follows the distribution of supernova remnants [13],

$$
n(r)=\left(r / R_{\odot}\right)^{0.7} \exp \left[-3.5\left(r-R_{\odot}\right) / R_{\odot}\right],
$$

with $R_{\odot}=8.5 \mathrm{kpc}$ as the distance of the Sun to the Galactic center.

Grammage - An important constraint on CR propagation models comes from ratios of stable primaries and secondaries produced by $\mathrm{CR}$ interactions on gas in the Galactic disk. In particular, the $\mathrm{B} / \mathrm{C}$ ratio has been recently measured by the AMS-02 experiment up to $670 \mathrm{GeV} /$ nucleon 14]. Above $E \gtrsim 10 \mathrm{GeV}$, it is consistent with a straight power-law.

Using the leaky-box formalism, various propagation models including plain diffusion, diffusion with convection and reacceleration were fitted in Ref. 15] to earlier data. In all cases, the grammage traversed by CRs at reference energies $E_{0} / Z=5-15 \mathrm{GeV}$ was found to lie in the range $9-14 \mathrm{~g} / \mathrm{cm}^{2}$. In their model with reacceleration, the grammage decreases as $X(E)=X_{0}\left(E / E_{0}\right)^{-0.3}$ above energies $E_{0}>20-30 \mathrm{GeV}$ per nucleon, while the best-fit value for the normalization constant $X_{0}$ of the grammage was determined to be $X_{0}=9.4 \mathrm{~g} / \mathrm{cm}^{2}$.

We calculate the average grammage $\langle X\rangle=$ $N^{-1} c \sum_{i=1}^{N} \int d t \rho\left(\boldsymbol{x}_{i}(t)\right)$ by injecting $N$ cosmic rays according to the radial distribution (11) at $z=0$ in the Galaxy, and following their trajectories $\boldsymbol{x}_{i}(t)$ until they reach the edge of the Galaxy. As a model for the gas distribution in the Galactic disk, we use $n(z)=n_{0} \exp \left(-\left(z / z_{1 / 2}\right)^{2}\right)$ with $n_{0}=0.3 / \mathrm{cm}^{3}$ at $R_{\odot}$ and $z_{1 / 2}=0.21 \mathrm{kpc}$, inspired by [16]. We set $n=10^{-4} \mathrm{~g} / \mathrm{cm}^{3}$ as minimum gas density up to the edge of the Milky Way at $|z|=10 \mathrm{kpc}$. Since the grammage $X(E) \propto E^{-\delta}$ scales as the confinement time $\tau(E) \propto E^{-\delta}$, we can use this quantity also as an indicator for changes in the CR intensity induced by a variation of the CR leakage rate.

In Fig. 11 we show the grammage traversed by CRs, with energies $E / Z$ between $10^{14} \mathrm{eV}$ and $10^{17} \mathrm{eV}$, propagated in the GMF model of Ref. [10]. The upper (green) line corresponds to computations using both the regular and the turbulent fields proposed in [10], while for the lower (red) curve we rescaled the turbulent field strength by a factor $0.1\left(B_{\mathrm{rms}} \rightarrow B_{\mathrm{rms}} / 10\right)$. The two lowestenergy data points shown here are consistent with the $X(E) \propto E^{-1 / 3}$ behavior expected for a turbulent magnetic field with a Kolmogorov power-spectrum. Around a few $\mathrm{PeV}$, the grammage steepens to an approximate

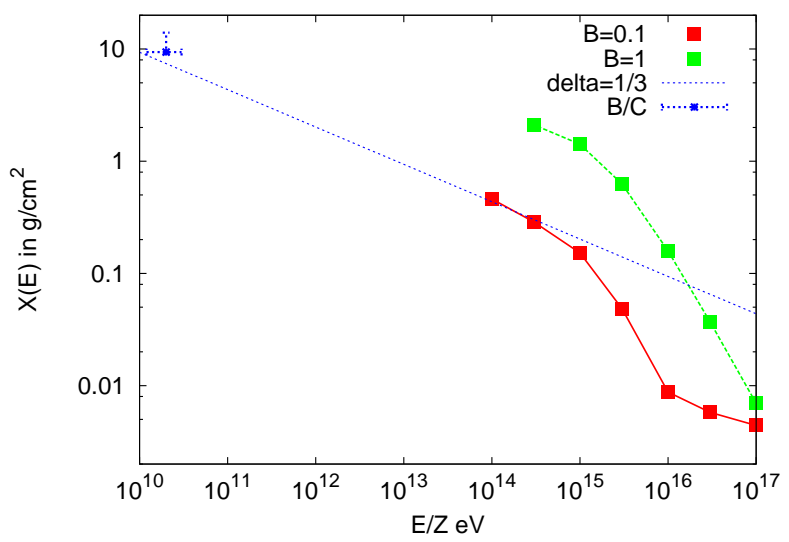

FIG. 1: Grammage $X(E)$ traversed by CR protons as a function of energy $E / Z$ for two different levels of magnetic turbulence in the GMF model of Ref. [10], with $l_{\mathrm{c}}=2 \mathrm{pc}$.

power-law $X(E) \propto E^{-1.3}$ which lies in-between the expectations for Hall diffusion $\left(X(E) \propto E^{-1}\right)$ and smallangle scattering $\left(X(E) \propto E^{-2}\right)$. The transition energy agrees well with the theoretical expectation: A steepening of the grammage is expected at the characteristic energy $E_{c}$ where the Larmor radius $r_{\mathrm{L}}$ equals the coherence length $l_{c}$. For $l_{c}=2 \mathrm{pc}$ and $B \approx 3 \mu \mathrm{G}$, the value of the critical energy is $E_{c} / Z \approx 6 \times 10^{15} \mathrm{eV}$, which is only slightly higher than what we find numerically. Finally, the turnover of the grammage which is visible in the lower curve at the highest energies corresponds to the approach of its asymptotic value obtained for straight line propagation in the limit $E \rightarrow \infty$. As a consequence, the predicted $\mathrm{CR}$ spectra above $E / Z \simeq 10^{16} \mathrm{eV}$ should harden by approximately one power, $\Delta \delta \sim 1$, using the model of Ref. [10] with a reduced turbulent field.

In addition to the data points, we show in Fig. 1 with a dotted (blue) line the extrapolation of the grammage to lower energies, assuming that $X(E) \propto E^{-1 / 3}$ as expected for a Kolmogorov power-spectrum. Based on this extrapolation, the GMF model [10] with full turbulence leads to a grammage at $E \lesssim 100 \mathrm{GeV}$ which is a factor $\sim 10$ above the determinations from e.g. B/C measurements (blue cross). This discrepancy is in line with our determination of the diffusion coefficient in a purely turbulent magnetic field with strength $B_{\mathrm{rms}}=4 \mu \mathrm{G}$ [17], which also disagreed by an order of magnitude with the extrapolation of the diffusion coefficient phenomenologically determined from the ratios of secondary to primary nuclei. Consistency with these measurements could be achieved, if the energy density of the turbulent magnetic field is reduced [31]. Such a rescaling is displayed as the lower (red) line in Fig. 11 In the following, we consider this case 32].

We have calculated the grammage also in the GMF model of Ref. [11]. The CR confinement time was found to be twice as large as for the GMF model of Ref. [10], 


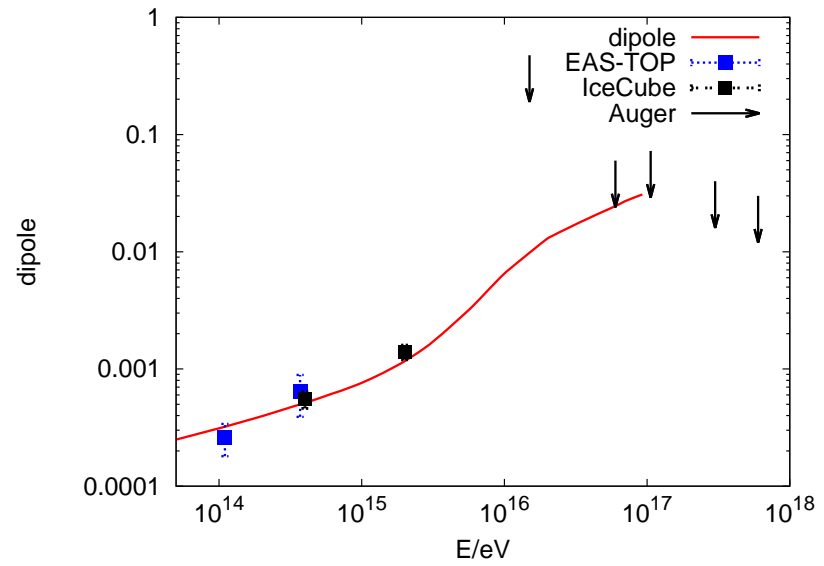

FIG. 2: Dipole amplitude $d(E)$ as a function of energy $E / Z$ in the GMF model of Ref. [10] with reduced turbulent field and $l_{\mathrm{c}}=2 \mathrm{pc}$.

leading to a stronger discrepancy between the extrapolation of the grammage to low energies and its determinations.

Cosmic ray anisotropy - In the diffusion approximation, the CR dipole anisotropy $d$ is given by $\boldsymbol{d}=$ $3 D \nabla \ln (n) / c$. The measurements or tight experimental upper limits on $d$ at high energies are typically difficult to reconcile with determinations of the diffusion coefficient at low energies, even for a Kolmogorov spectrum where $D(E) \propto E^{1 / 3}$. In our model, one expects the anisotropy to grow more rapidly above the knee. The diffusion coefficient scales there as $D \sim \frac{1}{3} l_{\mathrm{c}} c\left(R_{\mathrm{L}} / l_{\mathrm{c}}\right)^{\alpha}$, with $\alpha \approx 1.3$. We compute the average anisotropy and derive the energy dependence of $D(E)$ from the escape probability calculated previously, setting $D(E / Z) \propto \tau_{\text {esc }}(E / Z)$. We fix the proportionality constant by requiring that the dipole amplitude $d=\sum_{k} f_{k} d_{k}$ equals the dipole component $\tilde{d}$ observed by the EAS-TOP collaboration at $E=1.1 \times 10^{14} \mathrm{eV}$ [18]. $k$ labels the groups of nuclei we consider, $f_{k}$ is their fraction of the total CR flux, and $d_{k} \propto \tau_{\text {esc }}(E / Z)$ is their individual dipole.

In Fig. 2 we show the resulting dipole amplitude $d$ as a function of energy $E$. As expected, the amplitude raises below the knee as $E^{1 / 3}$, while it increases approximately as $E^{0.7}$ above. We also plot the values of $\tilde{d}$ observed by IceCube [19], as well as the $99 \%$ C.L. upper limits on $d_{\perp}$ from the Pierre Auger Observatory [20]. Comparing our estimate for the dipole amplitude with the upper limits at high energies, we conclude that the light component of the Galactic CR flux must be suppressed above $10^{17} \mathrm{eV}$. We expect the approximation $d \propto \tau_{\text {esc }}(E / Z)$ to break down for $E / Z \gtrsim 10^{17} \mathrm{eV}$ : Close to the semi-ballistic regime, a calculation of the anisotropy based on trajectories would be required, but is computationally extremely expensive.

Cosmic ray intensity - We now use the time-dependent intensity $I_{k}$ of various groups $k$ of CR nuclei as a test of our hypothesis that the knee is entirely explained by the energy-dependent CR leakage from the Milky Way. We distribute a discrete set of sources in the Galactic disk according to Eq. (1) and a fixed rate $\dot{N}$. Each source is assumed to inject the total energy $E_{\mathrm{p}}=1.0 \times 10^{50} \mathrm{erg}$ in CRs. Then the individual contributions $n_{i, k}(\boldsymbol{x}, t, E)$ from each source $i$ are added using a pre-calculated template in order to save computing time. We convert the total density $n_{k}\left(\boldsymbol{x}_{\odot}, t, E\right)$ at the position of the Sun into the predicted intensity $I_{k}\left(\boldsymbol{x}_{\odot}, t, E\right)=c /(4 \pi) n_{k}\left(\boldsymbol{x}_{\odot}, t, E\right)$ of the CR nuclei group $k$ as a function of time. Finally, we determine the relative fraction of energy transferred to the $k$.th group of nuclei and the exponent $\alpha_{k}$ of their injection spectrum $d N_{k} / d E \propto E^{-\alpha_{k}} \exp \left(-E / Z E_{c}\right)$ by a comparison of the predicted intensity to the measurements. We choose the energy $E_{c}$ above which we assume that the source spectrum is exponentially suppressed as $E / Z=10^{17} \mathrm{eV}$.

Note that $I_{k}(E, t)$ is predicted as a function of time. Since $I_{k}(E, t)$ fluctuates due to the discreteness of the sources, we show a $1 \sigma$ confidence band illustrating the spread around the predicted average intensity. In contrast, the grammage is measured at relatively low energies, $E \lesssim 1 \mathrm{TeV}$, where fluctuations due to the discrete source distribution play only a minor role and therefore only average values are relevant.

For the experimental data, we use above $E>10^{16} \mathrm{eV}$ the tabulated intensities of protons, helium, carbon (representing the CNO group), $\mathrm{Si}$ (for the $\mathrm{SiMg}$ group) and Fe nuclei (for Fe-Mg) from KASCADE-Grande data given in 2], while we employ in the energy range $E=$ $10^{15}-10^{16} \mathrm{eV}$ the KASCADE data read from Fig. 12 in 2]. Since the KASCADE and KASCADE-Grande proton data disagree in the overlapping energy range, we reduce the KASCADE-Grande proton flux by $30 \%$, and add this $30 \%$ proton flux to the He flux, in order to achieve agreement between the two data sets. Moreover, the discriminating power between $\mathrm{Si}$ and $\mathrm{Fe}$ in KASCADE was relatively poor and the absolute $\mathrm{Si}+\mathrm{Fe}$ abundance is small close to $10^{15} \mathrm{eV}$, and therefore we analyze these two groups of elements jointly 22, 23]. Finally, we determine the slope $\alpha_{k}$ and the normalisation of the intensities for individual CR groups with measurements at energies below the knee using the data from the CREAM experiment [24].

In the left panel of Fig. 3, we show our results (red solid points with error-bars) for the intensity of $\mathrm{p}, \mathrm{He}$, and the combined intensity of the MgSi and Fe groups compared to experimental data. Overall, we find that the assumptions used, a GMF model with rescaled Kolmogorov turbulence and $1 / E^{\alpha_{k}}$ injection spectra, lead to a consistent CR intensity in the full energy range $E \sim\left(10^{14}-10^{17}\right) \mathrm{eV}$ considered not only for protons, but also for He and heavy nuclei. Since CR escape depends only on rigidity, and since the exponents $\alpha_{k}$ are determined by the data below 

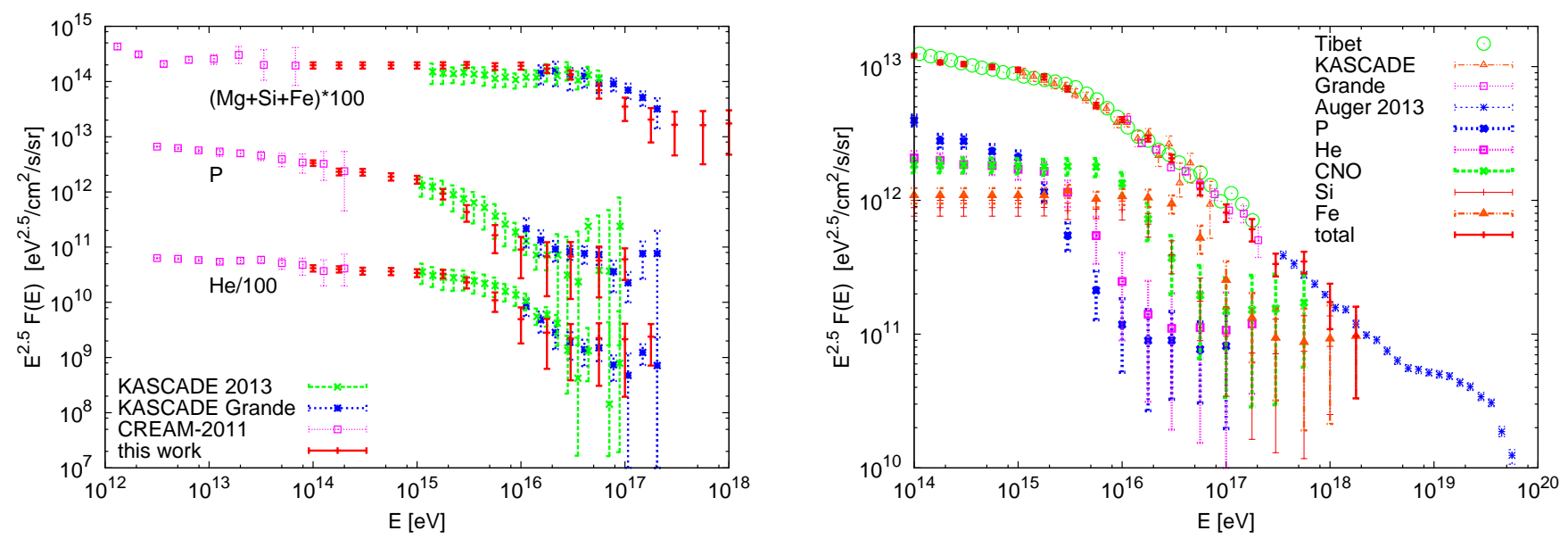

FIG. 3: Left: The (rescaled) intensity $I(E)$ of CR protons, He and Fe nuclei compared to the experimental data from KASCADE [2], KASCADE-Grande [2] and CREAM [24], using the rescaled turbulent GMF. Right: Intensity $I(E)$ of CR protons, $\mathrm{He}, \mathrm{CNO}, \mathrm{MgSi}$ and Fe nuclei as a function of energy $E$ per nucleus, obtained using the same GMF.

the knee, the relative shape of the different CR elements is fixed in this scheme. Note also that the recovery of the proton and helium spectra above $E / Z \sim 10^{16} \mathrm{eV}$ cannot be reproduced assuming power-law injection spectra and the full turbulent GMF of Ref. [10].

In the right panel of Fig. 3, we show the contribution of the CNO group and the resulting total CR intensity. The latter is compared to measurements of the total CR intensity by Tibet [25], KASCADE, KASCADE-Grande and Auger 26]: Because of the rigidity-dependent energy cutoff, Galactic CRs are dominated at the highest energies by iron, while the total intensity is exponentially suppressed above $3 \times 10^{18} \mathrm{eV}$. Thus Galactic Fe could give a significant contribution to the total $\mathrm{CR}$ spectrum up to the ankle, being especially important for composition studies.

The obtained source rate $\dot{N} \sim 1 / 180$ yr is only a factor six smaller than the Galactic SN rate and makes a GRB origin of Galactic CRs unlikely. Taken at face value, these numbers would require that a large fraction of $\mathrm{SNe}$ can accelerate protons up to $E \sim 10^{17} \mathrm{eV}$.

Conclusions - The two main explanations for the knee are $i$ ) a change in the CR confinement time in the Galaxy when their Larmor radius starts to be of the order of the coherence length $l_{\mathrm{c}}$ of the interstellar turbulence, and ii) a change in the number of sources able to accelerate CRs above $\approx 4 \mathrm{PeV}$. We have shown that, if the coherence length in the Galactic disk is of the order of $l_{\mathrm{c}}=2 \mathrm{pc}$ as suggested by Refs. [12], the CR escape time $\tau_{\text {esc }}(E) \sim X(E)$ and as a consequence the total CR intensity steepens at the correct energy. Moreover, the resulting rigidity-dependent knees in the individual intensities of the considered CR groups (p, He, CNO, and $\mathrm{MgSiFe}$ ) agree well with measurements.

In contrast, the change in the slope of $X(E / Z) \sim$ $\tau(E / Z)$ would be shifted to energies above $E / Z \simeq$
$10^{16} \mathrm{eV}$ for a coherence length $l_{\mathrm{c}}$ of the order of $l_{\mathrm{c}}=50 \mathrm{pc}$ in the Galactic disk. In this case, the knee would have to be explained by the possibility $i i$ ), yielding precious information on Galactic CR accelerators. More measurements of the coherence length of the turbulence as expected from e.g. SKA [27] will solve this crucial question.

A large coherence length $l_{\mathrm{c}}$ would worsen the tension between our computations of the grammage in GMF models like [10, 11] and its determination from B/C at low energies. In this Letter, we have therefore considered the possibility that the average strength of the turbulent magnetic field is reduced by a factor $\simeq 5-10$. In this case, we found agreement between our calculation of the escape time $\tau_{\text {esc }}$ and determinations of the grammage $X(E)$ at lower energies from the $\mathrm{B} / \mathrm{C}$ ratio. More importantly, the turnover of the grammage $X(E)$ leads to a hardening of the intensity of the nuclei with charge $Z$ around $E / Z \simeq 10^{16} \mathrm{eV}$. This energy behavior is very similar to the one seen in KASCADE-Grande measurements [1]: As a result, the observed energy dependence of various groups of CR nuclei can be explained in the energy range between $10^{14}$ and $10^{17} \mathrm{eV}$ as the modulation of power-law injection spectrum via CR leakage from the Galaxy.

The source rate in our scenario is relatively large, $\dot{N} \sim 1 / 180$ yr, and requires that a large fraction of $\mathrm{SNe}$ can accelerate protons up to $E \sim 10^{17} \mathrm{eV}$. Our estimate for the dipole anisotropy suggests on the other hand that CRs with energies beyond $E / Z \sim 10^{17} \mathrm{eV}$ are not predominantly Galactic, which requires an early transition to extragalactic CRs, before the ankle as e.g. in the dip model 28]. A hint about this is also given by KASCADEGrande [29]. Finally, we note that the suggested weakness of the turbulent GMF would facilitate the search for ultra-high energy CR sources by a smaller spread of their images on the sky [30]. 
We thank P. Blasi for raising the grammage question, and S. Ostapchenko and A. Strong for useful discussions. We are grateful to A. Haungs for advice about the KASCADE data. GG acknowledges funding from the European Research Council (Grant agreement No. 247039). The work of DS was supported in part by grant RFBR \# 13-02-12175-ofi-m.

[1] W. D. Apel et al. [KASCADE-Grande Collaboration], Phys. Rev. Lett. 107, 171104 (2011); Astropart. Phys. 36, 183 (2012).

[2] W. D. Apel et al. [KASCADE-Grande Collaboration], Astropart. Phys. 47, 54 (2011).

[3] R. Abbasi et al. [IceCube Collaboration], Astropart. Phys. 42, 15 (2013); M. G. Aartsen et al. [IceCube Collaboration], Phys. Rev. D 88, 042004 (2013).

[4] T. Stanev, P. L. Biermann and T. K. Gaisser, Astron. Astrophys. 274, 902 (1993); A. M. Hillas, J. Phys. G 31, R95 (2005).

[5] A. D. Erlykin and A. W. Wolfendale, J. Phys. G 23, 979 (1997); Astropart. Phys. 22, 47 (2004).

[6] For a work in this direction see e.g. A. R. Bell, K. Schure, B. Reville and G. Giacinti, Mon. Not. Roy. Astron. Soc. 431, 415 (2013); see also K. Kobayakawa, Y. Sato and T. Samura, Phys. Rev. D 66, 083004 (2002); L. G,. Sveshnikova, Astron. Astrophys. 409, 799 (2003); V. S. Ptuskin and V. N. Zirakashvili, Astron. Astrophys. 429, 755 (2005).

[7] V. L. Ginzburg and S. I. Syrovatskii, The Origin of Cosmic Rays (Pergamon Press, 1964).

[8] V. S. Ptuskin et al., Astron. Astrophys. 268, 726 (1993); J. Candia, E. Roulet and L. N. Epele, JHEP 0212, 033 (2002); J. Candia, S. Mollerach and E. Roulet, JCAP 0305, 003 (2003).

[9] G. Giacinti, M. Kachelriess, D. V. Semikoz and G. Sigl, JCAP 1207, 031 (2012).

[10] R. Jansson and G. R. Farrar, Astrophys. J. 757, 14 (2012); Astrophys. J. 761, L11 (2012).

[11] M. S. Pshirkov, P. G. Tinyakov, P. P. Kronberg and K. J. Newton-McGee, Astrophys. J. 738, 192 (2011); M. S. Pshirkov, P. G. Tinyakov and F. R. Urban, Mon. Not. Roy. Astron. Soc. 436, 2326 (2013).

[12] M. Iacobelli et al., Astron. Astrophys. 558, A72 (2013); see also G. Bernardi, A. G. de Bruyn, G. Harker et al., Astron. Astrophys. 522, 67 (2010); A. Ghosh, J. Prasad, S. Bharadwaj, S. S. Ali, J. N. Chengalur, Mon. Not. Roy. Astron. Soc. 426, 3295 (2012).

[13] D. A. Green, to appear in proc. IAU Symposium 296, "Supernova environmental impacts" (Eds. Ray and Mc-
Cray) [arXiv:1309.3072 [astro-ph.HE]].

[14] A. Oliva et al. [AMS-02 Collaboration], to appear in proc. of the 33rd ICRC (2013) [ID 1266].

[15] F. C. Jones et al., Astrophys. J. 547, 264 (2001).

[16] H. Nakanishi and Y. Sofue, Publ. Astron. Soc. Jap. 55, 191 (2003); Publ. Astron. Soc. Jap. 58, 841 (2006); see also the comparison in C. Evoli, D. Grasso and L. Maccione, JCAP 0706, 003 (2007).

[17] G. Giacinti, M. Kachelriess and D. V. Semikoz, Phys. Rev. Lett. 108, 261101 (2012).

[18] M. Aglietta et al. [EAS-TOP Collaboration], Astrophys. J. 692, L130-L133 (2009).

[19] R. Abbasi et al. [IceCube Collaboration], Astrophys. J. 746, 33 (2012).

[20] P. Abreu et al. [Pierre Auger Collaboration], Astrophys. J. 762, L13 (2012); I. Sidelnik et al. [Pierre Auger Collaboration], to appear in proc. of the 33rd ICRC (2013).

[21] Note that the experimental values $\tilde{d}$ are connected to the true dipole amplitude $d$ as $\tilde{d}=d_{\perp} /\langle\cos (\delta)\rangle$, where $d_{\perp}$ is the dipole component in the equatorial plane and $\langle\cos (\delta)\rangle$ the average declination of the events used in the harmonic analysis based only on R.A.; See J. Aublin and E. Parizot, Astron. Astrophys. 441, 407 (2005).

[22] A. Haungs, private communication.

[23] T. Antoni et al. [KASCADE Collaboration], Astropart. Phys. 24, 1 (2005).

[24] Y. S. Yoon et al., Astrophys. J. 728, 122 (2011).

[25] M. Amenomori et al. [TIBET III Collaboration], Astrophys. J. 678, 1165 (2008).

[26] A. Aab et al. [Pierre Auger Collaboration], to appear in proc. of the 33rd ICRC (2013) arXiv:1310.4620 [astroph.HE]].

[27] C. Carilli, S. Rawlings (Eds.), Science with the $S K A$, New Astron. Rev. 48, 979 (2004).

[28] V. S. Berezinsky, S. I. Grigorieva and B. I. Hnatyk, Astropart. Phys. 21, 617 (2004); V. Berezinsky, A. Z. Gazizov and S. I. Grigorieva, Phys. Lett. B 612, 147 (2005); Phys. Rev. D 74, 043005 (2006); R. Aloisio, V. Berezinsky, P. Blasi, A. Gazizov, S. Grigorieva and B. Hnatyk, Astropart. Phys. 27, 76 (2007).

[29] W. D. Apel et al., Phys. Rev. D 87, 081101 (2013).

[30] G. Giacinti, M. Kachelrieß, D. V. Semikoz and G. Sigl, Astropart. Phys. 35, 192 (2011).

[31] The GMF model of Ref. [10] is currently being revised, taking into account correlations between the electron density and the magnetic field. As a result, the turbulent field will be reduced by a factor 5-6 (G. Farrar, private communication).

[32] We have also performed calculations for $l_{c}=5 \mathrm{pc}$. In this case, the turbulent field has to be rescaled only by a factor 0.2 . 